\title{
Isolated dissection of the superior mesenteric artery: a case report and literature review
}

\author{
Aurelio Seidita, ${ }^{1}$ Filippo Barbiera, ${ }^{2}$ Elena Murmura, ${ }^{2}$ Maria Accardi, ${ }^{3}$ Alberto D’Alcamo, ${ }^{1}$ Giusi Randazzo, ${ }^{1}$ \\ Pasquale Mansueto, ${ }^{1}$ Antonio Carroccio, ${ }^{3}$ Delia Sprini ${ }^{1}$ \\ ${ }^{1}$ Internal Medicine, Department of Internal and Specialist Medicine, University Hospital of Palermo; ${ }^{2}$ Radiology, Hospital of \\ Sciacca, ASP Agrigento; ${ }^{3}$ Internal Medicine, Hospital of Sciacca, ASP Agrigento, Italy
}

\begin{abstract}
Isolated dissection of the superior mesenteric artery is rare; it is predominantly observed in men with the highest incidence in those over 40 years old. Contrast-enhanced multi-detector computed tomography is considered essential for the diagnosis, therapeutic management and follow-up. The therapeutic approach ranges from conservative medical treatment to surgery or endovascular stent placement, but there are, to date, no approved guidelines. We report the case of a 68 -year old man who entered our Emergency Department just for mild abdominal pain, which later proved to be due to acute dissection of the superior mesenteric artery.
\end{abstract}

\section{Introduction}

Superior mesenteric artery (SMA) dissection is a rare condition, especially if isolated. Given the lack of clinical information on this condition, the therapeutic approach is, to date, far from being standardized. Despite its rarity, SMA dissection may be one of the most important causes of acute intestinal ischemia. ${ }^{1,2}$ The clinical features of SMA dissection can be extremely variable, ranging from a completely asymptomatic condition, up to forms characterized by real acute abdomen. No laboratory tests help the diagnosis, while the more modern imaging techniques, in particular, contrast-en-

Correspondence: Aurelio Seidita, Dipartimento di Medicina Interna e Specialistica (DIMIS), via del Vespro 141, 90127 Palermo, Italy.

Tel.: +39.91.6552916 - Fax: +39.91.6552868.

E-mail: aurelio.seidita@unipa.it

Key words: superior mesenteric artery dissection, acute abdomen, mesenteric ischemia.

Contributions: AS, PM, AC, and DS, case management and review of the literature; FB and EM, image acquisition, analysis and interpretation; MA, AD'A, and GR, case management and acquisition of data.

Received for publication: 28 March 2013.

Revision received: 24 April 2013.

Accepted for publication: 27 May 2013.

This work is licensed under a Creative Commons Attribution NonCommercial 3.0 License (CC BY-NC 3.0).

(C) Copyright A. Seidita et al., 2014

Licensee PAGEPress, Italy

Italian Journal of Medicine 2014; 8:56-63

doi:10.4081/itjm.2013.168 hanced multidetector computed tomography (MDCT), grant high reliability for a definite diagnosis. ${ }^{3}$

Here we describe the case of a 68-year-old man who arrived at the Emergency Department of Giovanni Paolo II Hospital of Sciacca (AG) complaining only of mild abdominal pain, which later proved, according to radiological findings, to be acute SMA dissection.

\section{Case Report}

A 68-year old man was admitted to the Emergency Department of Giovanni Paolo II Hospital of Sciacca (AG) in August 2011 because of the sudden onset of mild abdominal pain. He reported a medical history of hypertension, under treatment with candesartan, irritable bowel syndrome and recurrent episodes of acute diverticulitis, being treated with periodic cycles of rifaximin; he also reported smocking about 15 cigarettes/day for the previous 30 years.

On physical examination the patient was restless, with a respiratory rate of 70 breaths per minute, a heart rate of 80 beats per minute in sinus rhythm, high blood pressure $(160 / 100 \mathrm{mmHg})$, diffuse abdominal mild pain (stronger in the right iliac fossa and left hip) and torpid peristalsis, without signs of peritonitis (Blumberg's sign negative). He underwent routine examinations, which were within normal range, except for the white blood cell count $\left(11.6 \times 10^{9} / \mathrm{L}\right.$; reference rage, 4.3 to $\left.10.8 \times 10^{9} / \mathrm{L}\right)$ and C-reactive protein level $(2.60 \mathrm{mg} / \mathrm{L}$; reference range $<0.5 \mathrm{mg} / \mathrm{L}$ ). The chest X-ray was normal, whereas abdominal radiographs revealed small airfluid levels in lower abdominal quadrants. Urinary tract ultrasound examination showed the presence of cysts in both kidneys (the largest in the right kidney, of about 1.5 $\mathrm{cm}$, the homologous one in the left kidney, of about 9 $\mathrm{cm})$. Finally contrast-enhanced MDCT of the abdomen 
was required because of suspected gastrointestinal (cecal) neoplasm. The contrast agent used for intravenous injection was iodixanol, a water-soluble nonionic contrast medium (containing $320 \mathrm{mg}$ of iodine per milliliter). An 18-gauge needle with cannula was used to administered $120 \mathrm{~mL}$ of iodixanol intravenously, followed by $40 \mathrm{~mL}$ of normal saline, using a dual headed pressure injector at a flow rate of $3.5 \mathrm{~mL} / \mathrm{sec}$. Arterial and venous phase images were obtained using the bolus tracking technique, with the region of interest in the abdominal aorta at the level of the celiac artery (threshold $=150 \mathrm{HU}$ ). Finally, sagittal, coronal and curved multiplanar reconstructions were made. MDCT showed that the diameter of the SMA was enlarged $(12 \mathrm{~mm})$ and identified the vessel's true and false lumina by the presence of an intimal flap just $15 \mathrm{~mm}$ after the origin of the SMA (Figures 1 and 2). The dissection extended anterogradely for $46 \mathrm{~mm}$ (Figure 3) and a modest amount of fluid at the origin of SMA was detected. There were no signs of bowel ischemia or ascites.

The patient started therapy with enoxaparin 6000 IU once daily; after 1 month of this therapy, the MDCT showed no meaningful changes, with the exception of reabsorption of the previously reported modest amount of fluid at the origin of the SMA.

\section{Discussion}

Isolated dissection of the SMA dissection is an extremely rare condition (Table 1). Bauersfeld et al. documented the first case in $1947 .{ }^{4}$ To date, fewer than 100 cases have been reported. ${ }^{5,6}$ Patients with SMA dissection are predominantly men ( $88 \%$ of cases) and over 40 years old (range, $45-87$ years). ${ }^{7,8}$

The most frequently reported risk factors include hypertension, atherosclerosis, fibromuscular dysplasia, cystic medial necrosis, and abdominal aortic aneurysm (Table 1); however, some dissections appear to occur spontaneously, without any identifiable etiology. ${ }^{9}$

Solis et al. hypothesized that dissection usually begins $1.5-3 \mathrm{~cm}$ from the orifice of the SMA, thus sparing the origin of the artery. This segment of SMA corresponds with the exit of the artery from the pancreas, exactly where it is exposed to shearing forces, because this area represents the border zone between the fixed retropancreatic portion and the more mobile distal mesenteric one. ${ }^{10,11}$

The natural history of the disease is unclear and differs from case to case. Most patients present with acute epigastric pain, probably due to the dissection itself or subsequent intestinal ischemia, sometimes associated with nausea, vomiting, abdominal distention, and melena. In some cases, patients present with chronic abdominal pain, sometimes related to food intake. Furthermore, patients may, occasionally, be completely asymptomatic. ${ }^{12}$
The natural history is unpredictable, and spontaneous resolution, definitive occlusion, aneurysm formation, or rupture may occur. The prognosis depends on the extent of the involvement of sub-segmental branches. However, isolated SMA dissection has his-

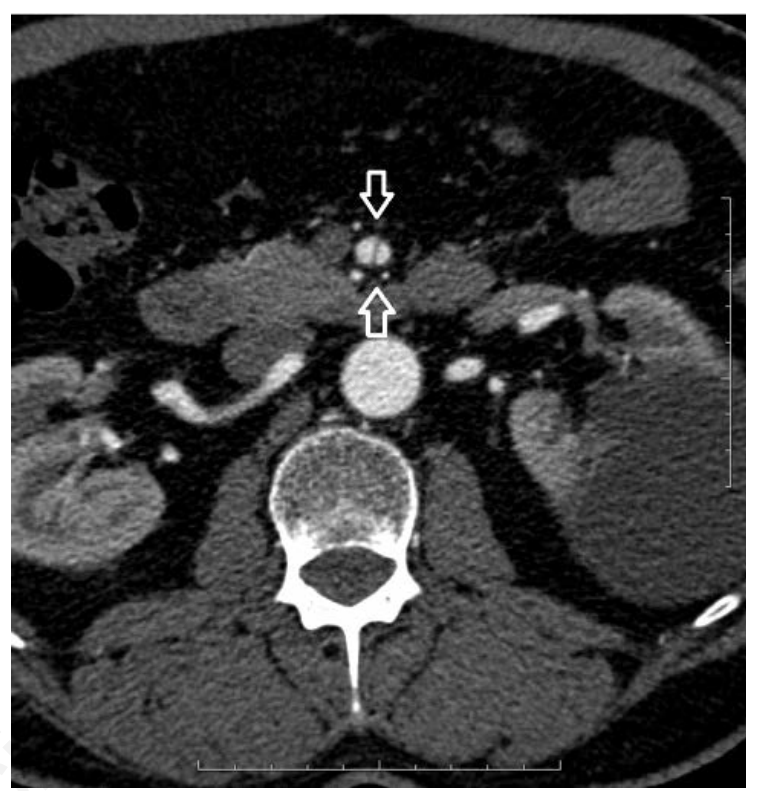

Figure 1. A contrast-enhanced computed tomography scan showing the enlarged diameter of the superior mesenteric artery (SMA). The true lumen and false lumen can be identified by the presence of an intimal flap after the origin of the SMA (arrows).

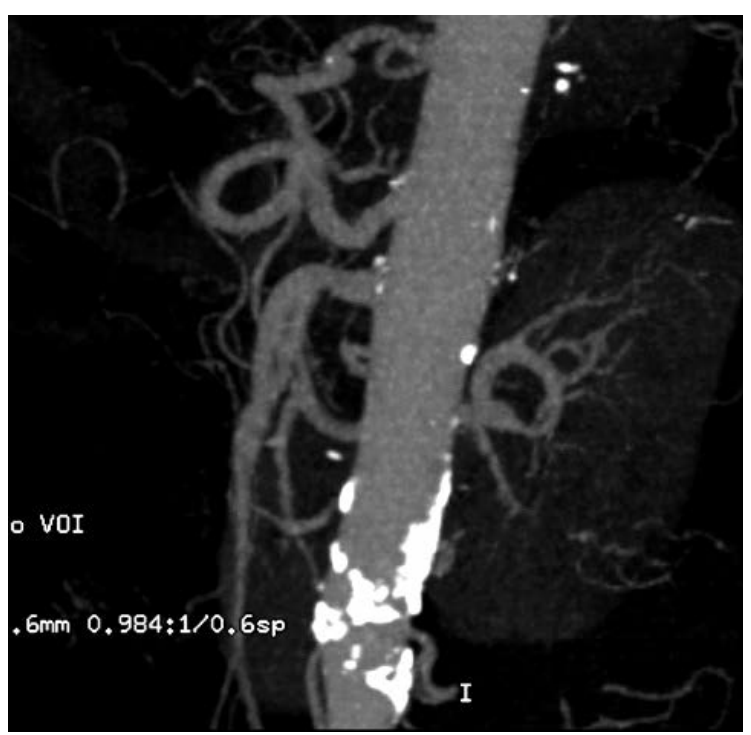

Figure 2. Maximum intensity projection reconstruction: the dissection starts just $\mathbf{1 5} \mathbf{~ m m}$ after the origin of the superior mesenteric artery. 
torically been considered to have a poor prognosis. Acute signs of intestinal ischemia or bleeding are poor prognostic features. ${ }^{13}$

Complications of SMA dissection include bowel infarction, intra-abdominal hemorrhage, acute peritonitis, shock, and late complications, such as uremia. ${ }^{14,15}$

Laboratory tests and abdominal radiography are usually unremarkable. Sometimes, laboratory tests show slightly elevated serum amylase levels, due to occlusion of duodeno-pancreatic arcades. Abdominal echocolor Doppler can be useful for demonstrating and following up hemodynamic changes within the SMA, bowel movements, and signs of bowel ischemia, such as wall thickening and dilated intestinal loops. ${ }^{7-13}$ Angiography is currently the gold standard imaging technique for diagnosing SMA dissection. ${ }^{16}$ Recently, contrast-enhanced computed tomography (CT) has become the most reliable diagnostic modality; indeed, diagnosis in the acute stage has become possible as a result of advanced and increasing use of CT imaging techniques, ${ }^{17}$ such as MDCT, leading to multiplanar reconstruction and reconstruction imaging, ${ }^{18}$ and computed tomography angiography (CTA). ${ }^{19-23}$ MDCT usually shows that the diameter of the SMA is enlarged and that there is increased attenuation of the fat surrounding the artery itself, together with images of true and false lumina, which can be identified by the presence of an intimal flap after the origin of the SMA (Table 2). In our case, MDCT provided enough information to diagnose spontaneous SMA dissection, and angiography was not necessary. This technique was also useful for our patient's follow-up. Sakamoto et al. categorized SMA dissection into four types on contrast-enhanced CT scanning. ${ }^{24}$
Recently, Yun et al. devised a new classification based on angiographic findings: in this classification, there are three types, with two subtypes (Table 3). ${ }^{25}$ However, neither Sakamoto et al. nor Yun et al. found a clear relationship between radiological appearance and clinical course.

The differential diagnosis of SMA includes acute SMA embolism, acute SMA thrombosis, non-occlusive mesenteric ischemia, mesenteric venous thrombosis, and aortic dissection (Table 2) ${ }^{26}$

Acute SMA embolism accounts for approximately $40-50 \%$ of all episodes of acute mesenteric ischemia

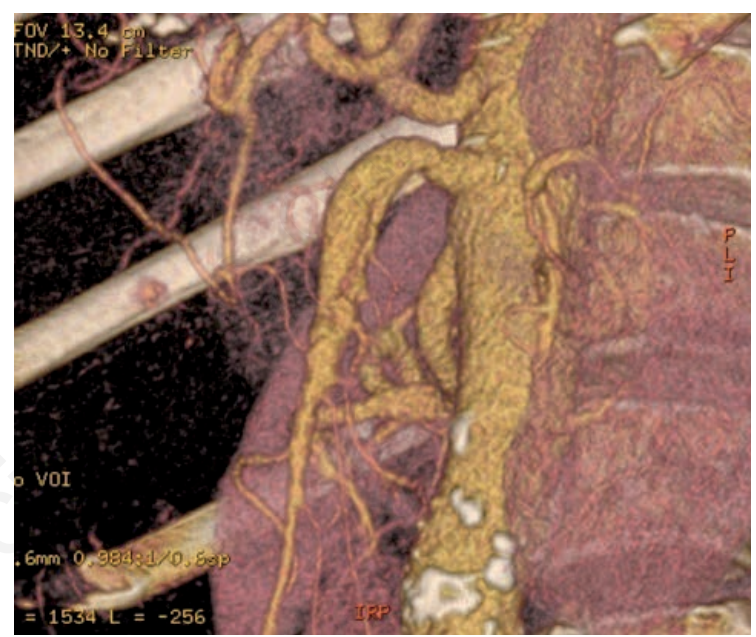

Figure 3. Virtual reconstruction showing the superior mesenteric artery dissection associated with an enlarged diameter of the artery.

Table 1. Summary of superior mesenteric artery dissection.

\begin{tabular}{ll}
\hline Etiology & $\begin{array}{l}\text { According to the hypothesis proposed by Solis et al., the dissection is thought to be caused by stress on the wall of } \\
\text { the artery at the inferior pancreatic edge }{ }^{21}\end{array}$ \\
\hline Incidence & Fewer than 100 cases have been reported in the literature since the first case described by Bauerfeld in 1947 \\
\hline Gender predilection & Patients are predominantly male ( $88 \%$ of cases) \\
\hline Age predilection & Range: $45-87$ years old \\
\hline Risk factors & $\begin{array}{l}\text { Hypertension, atherosclerosis, fibromuscular dysplasia, mycotic infections, trauma, connective tissue disorders, cystic } \\
\text { medial necrosis, abdominal aortic aneurysm, vasculitides such as giant cell arteritis, Takayasu's arteritis, polyarteritis } \\
\text { nodosa and iatrogenic dissections due to endovascular interventions; however, some dissections occur spontaneously } \\
\text { without any identifiable etiology }\end{array}$ \\
\hline Treatment & $\begin{array}{l}\text { Patients should initially receive conservative treatment (anticoagulation, CT follow-up) when the mesenteric blood } \\
\text { supply to intestines is not severely compromised by the dissection and the patients have no peritoneal signs } \\
\text { Patients who have recurrent symptoms after conservative treatment should undergo endovascular revascularization } \\
\text { if they have no peritonitis, or surgical intervention if peritonitis develops }\end{array}$ \\
\hline Prognosis & $\begin{array}{l}\text { The prognosis depends on the extent of involvement of sub-segmental branches. Acute signs of bleeding or ischemia } \\
\text { are poor prognostic features }\end{array}$ \\
\hline Findings on imaging & $\begin{array}{l}\text { MDCT shows an enlarged SMA diameter, increased attenuation of the fat around the SMA, true and false lumina } \\
\text { can be identified by the presence of an intimal flap after the origin of the SMA }\end{array}$ \\
\hline
\end{tabular}

CT, computed tomography; MDCT, multidetector computed tomography; SMA, superior mesenteric artery. 
Table 2. Differential diagnosis of superior mesenteric artery dissection.

\begin{tabular}{|c|c|c|c|}
\hline & CT & Angiography & Clinical findings \\
\hline SMA dissection & $\begin{array}{l}\text { MDCT shows an enlarged SMA } \\
\text { diameter, increased attenuation of the } \\
\text { fat around the SMA; true and false } \\
\text { lumina can be identified by the } \\
\text { presence of an intimal flap after } \\
\text { he artery origin }\end{array}$ & $\begin{array}{l}\text { The arteriogram demonstrates a } \\
\text { narrowed true lumen and an } \\
\text { aneurysmal dilated false lumen. The } \\
\text { arteriograms can reveal the intimal flap } \\
\text { and a reduced blood flow }\end{array}$ & $\begin{array}{l}\text { The most common symptom is acute } \\
\text { epigastric pain (caused by the } \\
\text { dissection itself or intestinal ischemia) } \\
\text { sometimes associated with nausea, } \\
\text { vomiting, melena, and abdominal } \\
\text { distention. In some cases, the patient } \\
\text { has chronic abdominal pain. } \\
\text { Additionally, patients may be } \\
\text { asymptomatic }\end{array}$ \\
\hline
\end{tabular}

Acute SMA embolism
CTA can show the acute occlusions as filling defects in the vessel lumen

No or only very few collateral vessels are present

In patients with prior embolic events, re-canalized vessels may be seen
The angiographic hallmark is abrupt termination of the vessel (cutoff sign)

Acute SMA embolism is often characterized by multiple occlusions
Most emboli in the SMA lodge just beyond the origin of the middle colic artery

Acute embolism is often associated with atrial fibrillation
Acute mesenteric Filling defect in the vessel lumen, more Angiography can show the acute artery thrombosis proximal than embolic occlusion

CTA can show these findings in addition to visualizing collateral vessels occlusion as a filling defect in the SMA, more proximal than embolic occlusion

Collateral vessels may be present

Typically associated with a pre-existing atherosclerotic lesion. In up to $50 \%$ of cases, a history of intestinal angina is present. The abdominal pain and other symptoms may be more insidious than those of embolic occlusion because of the development of a collateral circulation

The SMA is typically occluded within the first $2 \mathrm{~cm}$ from its origin, in contrast to acute embolic occlusions, which occur more distally

Non-occlusive The bowel wall of the involved mesenteric ischemia segments may be normal or thickened. Enhancement is variable: absent or diminished enhancement, increased enhancement, or halo or target-type enhancement. Fat stranding of the mesentery and ascites are visible
Angiography does not show vascular obstruction, but diffuse splanchnic vasospasm, narrowing of SMA branches, low flow and poor distal filling
Non-occlusive mesenteric ischemia usually develops during an episode of cardiogenic shock or a state of hypoperfusion in which excessive sympathetic activity results in secondary vasoconstriction of the mesenteric arteries

\section{Mesenteric \\ venous} thrombosis
Persistent, well-defined intraluminal filling defects with central low attenuation, which may be surrounded by well-defined, rim-enhancing venous walls

Accompanying collateral circulation, engorgement of mesenteric veins, and mesenteric edema may be present

Bowel wall thickening

Target sign resulting from submucosal edema or hemorrhage

The thickened bowel wall may appear to be hyperattenuated because of intramural venous engorgement
Mesenteric arteriography with delayed venous phase imaging may demonstrate venous occlusion or an intraluminal thrombus

Superior mesenteric arterial spasm is frequently observed, although prolongation of the arterial angiographic phase and other subtle findings may also result from superior mesenteric venous occlusion
Associated risk factors are portal hypertension, hypercoagulation, trauma, intra-abdominal inflammatory diseases and recent surgery. Many cases are idiopathic. Acute mesenteric ischemia develops when mesenteric vein thrombosis is associated with a lack of an adequate venous collateral circulation
The arteriogram demonstrates a narrowed true lumen and an aneurysmal dilated false lumen, the intimal flap and reduced blood flow clarifying the dissection, defining e and re-entry points, differentiating thrombus from slow flow, and evaluating the involvement of branch vessels
The main symptom is the pain

The most common classification systems of aortic dissection are the Standford (types A and B) and DeBakey (types I, II, III) systems

CT, computed tomography; SMA, superior mesenteric artery; MDCT, multidetector computed tomography; CTA, CT angiography. 
and is often associated with atrial fibrillation. Most SMA emboli lodge just beyond the origin of the middle colic artery. However, acute SMA embolism is often characterized by multiple occlusions. It usually presents with acute and massive abdominal pain, sometimes morphine-resistant, together with vomiting (71\%), diarrhea (42\%) and hematochezia (21\%). ${ }^{27}$ The angiographic and CTA hallmark of an embolic occlusion is the abrupt termination of the vessel (cutoff sign) (Table 1). ${ }^{28}$

Acute superior mesenteric artery thrombosis is typically associated with a preexisting atherosclerotic lesion and it has been estimated to be responsible for $20-30 \%$ of all cases of acute mesenteric ischemia. In up to $50 \%$ of cases, a history of intestinal angina is present. In contrast to the abrupt, catastrophic onset of symptoms associated with acute SMA embolism, abdominal pain and symptoms associated with acute mesenteric artery thrombosis may be more insidious, because of the development of collateral circulation. Usually this condition is associated with chronic pain spreading after meals, also known as angina abdominis. Angiography and CTA might show acute occlusion as a filling defect in the SMA, more proximally than in cases of embolism (Table 1). ${ }^{29}$

Non-occlusive mesenteric ischemia seems to be responsible for approximately $25 \%$ of cases of acute mesenteric ischemia, and the mortality rate associated with this condition has been reported to be as high as $70 \%$. It usually develops during cardiogenic shock or hypoperfusion states, in which excessive sympathetic activity results in secondary vasoconstriction of the mesenteric arteries. Hypoperfusion results in increased vascular permeability, which leads to extravascular leakage of plasma, red blood cells, or both into the bowel wall, the mesentery, and the peritoneal cavity. Shock bowel is a variety of non-occlusive mesenteric ischemia, caused by hypotensive shock induced by blunt abdominal trauma. Ischemic colitis and obstructive colitis may be considered similar clinical entities. No defined symptoms or signs can be attributed to nonocclusive mesenteric ischemia. There may be absolutely no early symptoms or symptoms ranging from abdominal discomfort to restlessness. Abdominal pain (reported to be absent in $23 \%$ of cases), bloody stools and progressive reduction of consciousness may then appear, leading, finally, to a picture of acute abdomen. ${ }^{30,31}$ Angiography does not show vascular obstruction, but diffuse splanchnic vasospasm (Table 1). There are few published reports of CTA findings, which usually showed normal mesenteric vessels (both arteries and veins), associated with bowel wall thickening and pneumatosis (i.e. gas in the bowel wall). However, among several forms of mesenteric ischemia, non-occlusive mesenteric ischemia is the most difficult condition to diagnose on CT, and angiography is often required for correct and confident diagnosis. ${ }^{32,33}$

Mesenteric vein thrombosis accounts for $5-15 \%$ of all cases of acute mesenteric ischemia. The most common associated risk factors include portal hypertension, coagulation disorders, and trauma (Table 1). However, many cases are idiopathic. Subsequent acute mesenteric ischemia might develop when mesenteric vein thrombosis is associated with a lack of an adequate venous collateral circulation, which results in the development of intestinal mucosal edema and arterial hypoperfusion. Clinically, at least three different pictures can be differentiated: acute, subacute and chronic. The acute picture usually involves spreading pain, hematochezia and reduction of peristalsis, leading to acute abdomen and shock. Subacute mesenteric vein thrombosis produces the same picture as the acute one, except for a smoother onset. In contrast, the chronic picture may be associated with non-specific symptoms or signs and, sometimes, can even be completely asymptomatic. ${ }^{34}$ Angiography, with delayed venous phase imaging, may demonstrate venous occlusion or an intraluminal thrombus. CT of mesenteric vein thrombosis shows persistent, well-defined intraluminal filling defects, with central low attenuation (Table 1). Diagnostic angiography is usually reserved for cases in which clinically suspected porto-mesenteric venous thrombosis cannot be established by noninvasive techniques. ${ }^{35}$

Approximately $5 \%$ of patients with aortic dissection develop acute mesenteric ischemia as a complication of the dissection process. Isolated dissections of the visceral arteries usually occur in association with cystic degeneration or as a complication of catheter angiography and are extremely rare. Tearing and ripping pain, which migrates in the direction of dissection, is the most important symptom of aortic

Table 3. Superior mesenteric artery angiographic classification.

\begin{tabular}{|c|c|}
\hline Type I & Patent true and false lumina that show entry and re-entry sites \\
\hline Type II & $\begin{array}{l}\text { Patent true lumen but no re-entry flow from the false lumen } \\
\text { IIa: visible false lumen but no visible re-entry site (blind pouch of false lumen) } \\
\text { IIb: no visible false luminal flow (thrombosed false lumen), which usually causes true luminal narrowing }\end{array}$ \\
\hline Type III & SMA dissection with occlusion of the SMA \\
\hline
\end{tabular}

SMA, superior mesenteric artery. Modified from Yun et al., 2009.16 
dissection, associated, usually, with nausea, sweating, shortness of breath, weakness and, sometimes, loss of consciousness. Several others organ-specific signs and symptoms may be connected with occlusion of the arteries which arise from the aorta. ${ }^{36}$ Arteriography demonstrates a narrowed true lumen, an aneurysmal dilated false lumen, an intimal flap and reduced blood flow. CTA is an excellent technique for assessing these patients, showing the dissection, defining entry and re-entry points, differentiating thrombus from slow flow, and evaluating branch vessel involvement. ${ }^{37}$

In the context of dissection of the SMA, acute bowel infarction is very rare, and most cases have slow progression to chronic ischemia. ${ }^{38,39}$ The most common CT manifestation of accompanying bowel ischemia is bowel wall thickening. This last, non-specific, finding may be identified from the target sign, with alternating intramural areas of high and low attenuation, resulting from submucosal edema or hemorrhage. The thickened bowel wall may appear to be hyperattenuated: this is assumed to be due to intramural venous engorgement. Other important $\mathrm{CT}$ findings include bowel enlargement and, less commonly, intestinal pneumatosis. These last two findings are more specific signs of ischemic bowel disease..$^{40}$

Some treatment algorithms for the management of spontaneous SMA dissection have been reported in literature, but, to date, there is no established, unanimous opinion about conservative medical management, endovascular therapy, or surgical revascularization. Some cases have been successfully treated by conservative therapy, such as anticoagulant drugs, ${ }^{41,42}$ but there is not enough experience to demonstrate that the efficacy and safety of this approach are superior to those of other approaches. However, most of the reports agree about the following therapeutic strategies: ${ }^{43}$ i) patients without imminent vessel rupture, symptoms of bowel ischemia or peritoneal signs: conservative treatment; ii) patients with an increased risk of perioperative complications, without severe mesenteric ischemia or peritonitis: percutaneous endovascular stent placement; iii) luminal thrombosis, increasing size of SMA aneurysmal dilatation, peritoneal signs, or persistent symptoms despite anticoagulation: open surgery (aorto-mesenteric or ilio-mesenteric bypass, thrombectomy, intimectomy, with or without patch angioplasty, ligation and resection). ${ }^{44,45}$ Conservative managements mainly include anticoagulation and blood pressure control. Nevertheless, all studies indicate that a non-operative approach with anticoagulant drugs often does not prevent disease progression, so close follow-up is strongly recommended. ${ }^{46}$ Early minimally invasive techniques, such as percutaneous endovascular stent placement, are indicated in cases with short segment dissection without bowel ischemia or signs of peritonitis and in patients not improving on conservative therapy and who have an increased risk of perioperative complications. It is an efficient and less aggressive approach, which provides excellent immediate results and may become a valuable option for mesenteric revascularization in the near future ${ }^{47}$ Finally, surgical methods, such as bypass with venous grafts and thrombectomy, are indicated in all cases with signs of bowel ischemia and perforation; they have been performed with good short-term results..$^{29,48}$

\section{Conclusions}

Isolated SMA dissection is a rare pathology, but it should be considered in the differential diagnosis of abdominal angina and acute abdomen. The clinician's suspicion can be confirmed by CT studies. MDCT shows that the diameter of the SMA is enlarged SMA and that there is increased attenuation of the fat around the SMA; it may also demonstrate a true lumen and false lumen by the presence of an intimal flap after the origin of the SMA. CT studies, i.e. MDCT, are essential not only for the diagnosis, but also for the therapeutic management and follow-up. There is no consensus on the best treatment of spontaneous isolated dissection of the SMA, and further information is required to establish optimal management guidelines for the disease. However, at present, patients should initially receive conservative treatment when the mesenteric blood supply to the intestines is not severely compromised by the dissection and the patients have no signs of peritonitis, as in our case report. When patients have recurrent symptoms after conservative treatment and no signs of peritonitis, endovascular repair for SMA revascularization is indicated, while surgical intervention is indicated when peritonitis occurs.

\section{References}

1. Yang HJ, Cho YK, Son TJ, et al. Rapidly aggravated dissecting flap by angiography during percutaneous stent placement for acute isolated superior mesenteric artery dissection. Yonsei Med J 2011;52:859-62.

2. Min SI, Yoon KC, Min SK, et al. Current strategy for the treatment of symptomatic spontaneous isolated dissection of superior mesenteric artery. J Vasc Surg 2011;54:461-6.

3. Katsura M, Mototake H, Takara H, et al. Management of spontaneous isolated dissection of the superior mesenteric artery: case report and literature review. World J Emerg Surg 2011;6:16.

4. Bauersfeld SR. Dissecting aneurysm of the aorta: a presentation of fifteen cases and a review of recent literature. Ann Intern Med 1947;26:873-89.

5. Subhas G, Gupta A, Nawalany M, et al. Spontaneous isolated superior mesenteric artery dissection: a case report and literature review with management algorithm. Ann Vasc Surg 2009;23:788-98. 
6. Miyamoto N, Sakurai Y, Hirokami M, et al. Endovascular stent placement for isolated spontaneous dissection of the superior mesenteric artery: report of a case. Radiat Med 2005;23:520-4.

7. Takayama T, Miyata T, Shirakawa M, et al. Isolated spontaneous dissection of the splanchnic arteries. J Vasc Surg 2008;48:329-33.

8. D'Ambrosio N, Friedman B, Siegel D, et al. Spontaneous isolated dissection of the celiac artery: CT findings in adults. AJR Am J Roentgenol 2007;188:W506-11.

9. Chang SH, Lien WC, Liu YP, et al. Isolated superior mesenteric artery dissection in a patient without risk factors or aortic dissection. Am J Emerg Med 2006;24:385-7.

10. Solis MM, Ranval TJ, McFarland DR, et al. Surgical treatment of superior mesenteric artery dissecting aneurysm and simultaneous celiac artery compression. Ann Vasc Surg 1993;7:457-62.

11. Katsura M, Mototake H, Takara H, et al. Management of spontaneous isolated dissection of the superior mesenteric artery: case report and literature review. World J Emerg Surg 2011;6:16.

12. Kang TL, Teich DL, McGillicuddy DC. Isolated, spontaneous superior mesenteric and celiac artery dissection: case report and review of literature. J Emerg Med 2011;40:e21-5.

13. Javerliat I, Becquemin JP, d'Audiffret A. Spontaneous isolated dissection of the superior mesenteric artery. Eur J Vasc Endovasc Surg 2003;25:180-4.

14. Vignati PV, Welch JP, Ellison L, et al. Acute mesenteric ischemia caused by isolated superior mesenteric artery dissection. J Vasc Surg 1992;16:109-12.

15. Shinji H, Yoshiharu H, Mitsui M, et al. Spontaneous and isolated dissection of the main trunk of the superior mesenteric artery. Ann Thoracic Cardiovasc Surg 2002;8:4.

16. Yun WS, Kim YW, Park KB, et al. Clinical and angiographic follow-up of spontaneous isolated superior mesenteric artery dissection. Eur J Vasc Endovasc Surg 2009;37:572-7.

17. Lalitha P, Reddy B. Isolated superior mesenteric artery dissection. Indian J Radiol Imaging 2010;20:132-4.

18. Saba L, Sanfilippo R, Atzeni M, et al. Superior mesenteric artery spontaneous and isolated dissection diagnosed by using MDCTA. Eur Rev Med Pharmacol Sci 2010;14: 235-8.

19. Suzuki S, Furui S, Kohtake H, et al. Isolated dissection of the superior mesenteric artery: CT findings in six cases. Abdom Imaging 2004;29:153-7.

20. Hyodoh H, Hyodoh K, Takahashi K, et al. Three-dimensional CT imaging of an isolated dissecting aneurysm of the superior mesenteric artery. Abdom Imaging 1996;21:515-6.

21. Sheldon PJ, Esther JB, Sheldon EL, et al. Spontaneous dissection of the superior mesenteric artery. Cardiovasc Intervent Radiol 2001;24:329-31.

22. Furukawa H, Moriyama N. Spontaneous dissection of the superior mesenteric artery diagnosed on multidetector helical CT. J Comput Tomogr 2002;26:143-4.

23. Yoo BR, Han HY, Cho YK, et al. Spontaneous rupture of a middle colic artery aneurysm arising from superior mesenteric artery dissection: diagnosis by color Doppler ultrasonography and CT angiography. J Clin Ultrasound 2012;40:255-9.

24. Sakamoto I, Ogawa Y, Sueyoshi E, et al. Imaging ap- pearances and management of isolated spontaneous dissection of the superior mesenteric artery. Eur J Radiol 2007;64:103-10.

25. Yun WS, Kim YW, Park KB, et al. Clinical and angiographic follow-up of spontaneous isolated superior mesenteric artery dissection. Eur J Vasc Endovasc Surg 2009;37:572-7.

26. Shih MC, Hagspiel KD. CTA and MRA in mesenteric ischemia: part 1. Role in diagnosis and differential diagnosis. AJR Am J Roentgenol. 2007;188:452-61.

27. Acosta S, Björck M. Acute thrombo-embolic occlusion of the superior mesenteric artery: a prospective study in a well defined population. Eur J Vasc Endovasc Surg 2003;26:179-83.

28. Savassi-Rocha PR, Veloso LF. Treatment of superior mesenteric artery embolism with a fibrinolytic agent: case report and literature review. Hepatogastroenterology 2002;49:1307-10.

29. Gupta R, Chimpiri AR, Saucedo JF. Superior mesenteric artery thrombosis managed percutaneously by timely combining aspiration thrombectomy with angioplasty and stents. J Thromb Thrombolysis 2010;29:105-7.

30. Kazui T, Yamasaki M, Abe K, et al. Non-obstructive mesenteric ischemia: a potentially lethal complication after cardiovascular surgery: report of two cases. Ann Thorac Cardiovasc Surg 2012;18:56-60.

31. Howard TJ, Plaskon LA, Wiebke EA, et al. Nonocclusive mesenteric ischemia remains a diagnostic dilemma. Am J Surg 1996;171:405-8.

32. Björck M, Wanhainen A. Nonocclusive mesenteric hypoperfusion syndromes: recognition and treatment. Semin Vasc Surg 2010;23:54-64.

33. Wildermuth S, Leschka S, Alkadhi H, et al. Multislice $\mathrm{CT}$ in the pre- and post-interventional evaluation of mesenteric perfusion. Eur Radiol 2005;15:1203-10.

34. Kumar S, Kamath PS. Acute superior mesenteric venous thrombosis: one disease or two? Am J Gastroenterol 2003;98:1299-304.

35. Harnik IG, Brandt LJ. Mesenteric venous thrombosis. Vasc Med 2010;15:407-18.

36. Fuster V, Ip JH. Medical aspects of acute aortic dissection. Semin Thorac Cardiovasc Surg 1991;3:219-24.

37. Sandridge L, Kern JA. Acute descending aortic dissections: management of visceral, spinal cord, and extremity malperfusion. Semin Thorac Cardiovasc Surg 2005; 17:256-61.

38. Wu B, Zhang J, Yin MD, et al. Isolated superior mesenteric artery dissection: case for conservative treatment and endovascular repair. Chin Med J 2009;122:238-40.

39. Kaliappan GS, Saravanan S, Premnath U, et al. Spontaneous isolated superior mesenteric artery dissection - report of two cases. J Radiol Case Rep 2009;3:6-13.

40. Florian A, Jurcut R, Lupescu I, et al. Mesenteric ischemia-a complex disease requiring an interdisciplinary approach. A review of the current literature. Rom J Intern Med 2010;48:207-22.

41. Nagai T, Torishima R, Uchida A, et al. Spontaneous dissection of the superior mesenteric artery in four cases treated with anticoagulation therapy. Intern Med 2004;43:473-8.

42. Takayama H, Takeda S, Saitoh SK, et al. Spontaneous isolated dissection of the superior mesenteric artery. Intern Med 2002;41:713-6. 
43. Buchs NC, Charbonnet P, Schwenter F, Becker CD, et al. Spontaneous dissection of the superior mesenteric artery and the right hepatic artery: a case report. J Med Case Rep 2010;16:87.

44. Zhang X, Sun Y, Chen Z, et al. Therapeutic regimen options for isolated superior mesenteric artery dissection. Vasc Endovascular Surg 2012;46:277-82.

45. Min SI, Yoon KC, Min SK, et al. Current strategy for the treatment of symptomatic spontaneous isolated dissection of superior mesenteric artery. J Vasc Surg 2011; 54:461-6.
46. Yasuhara H, Shigematsu H, Muto T. Self-limited spontaneous dissection of the main trunk of the superior mesenteric artery. J Vasc Surg 1998;27:776-9.

47. Leung DA, Schneider E, Kubik-Huch R, et al. Acute mesenteric ischemia caused by spontaneous isolated dissection of the superior mesenteric artery: treatment by percutaneous stent placement. Eur Radiol 2000;10:1916-9.

48. Cho BS, Lee MS, Lee MK, et al. Treatment guidelines for isolated dissection of the superior mesenteric artery based on follow-up CT findings. Eur J Vasc Endovasc Surg 2011;41:780-5. 